\title{
QUASTCYCLIC LIGHT CHANGES IN THREE LOW MASS T TAURI STARS
}

H. Mauder

Astronomisches Institut / Universität Tübingen, 7400 Tübingen, West Germany

During a photographic survey of the Chamaeleon T association in 1971/1972, evidence was found for quasiperiodic light changes of three variable stars, see Mauder and Sosna (1975). The period of 6.2 days for SY Cha is well seen, the periods of 7 days for $\mathrm{VZ}$ Cha and of 8 days for TW Cha are less pronounced. Intrinsic variations are present in addition to the cyclic variations. The three stars were classified by Hoffmeister (1963) as T Tauri type stars from their light variations. Objective prism spectra obtained by Henize and Mendoza (1973) confirm this classification, they found conspicuous emission lines. For SY Cha and TW Cha they got slit spectra, too, which show the typical veiling. The stars SY Cha, IW Cha and VZ Cha have been observed in the UBV system from 1974 March 12 until 1974 March 22, using the ESO standard photometer. In Figures 1 - 3 the light and colour curves are given for SY Cha, TW Cha and VZ Cha. Each point is a mean of generally 8 to 12 integrations, each integration lasting 5 seconds.

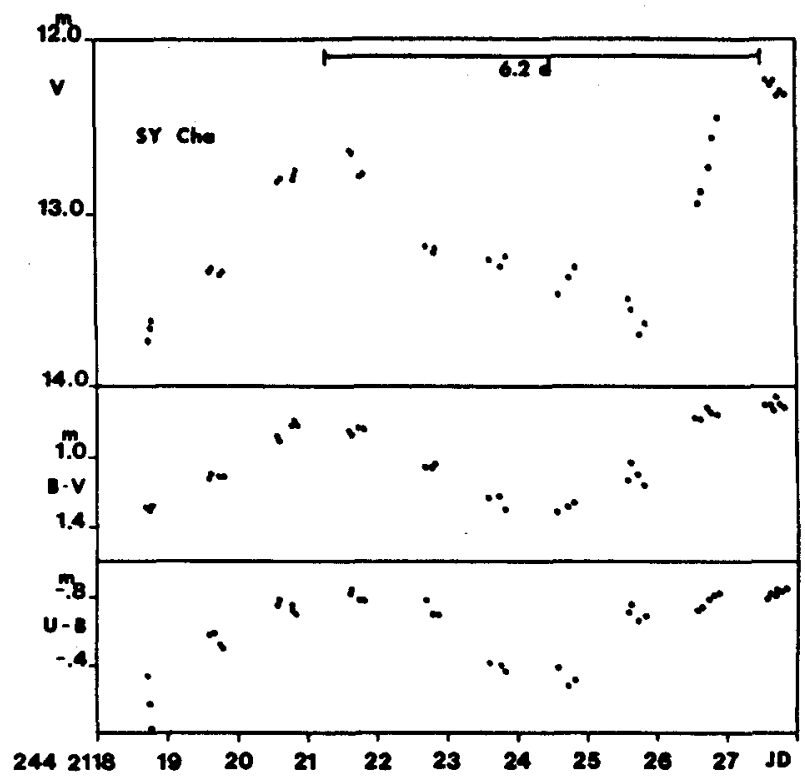

Figure 1: Light and colour curves of SY Cha 


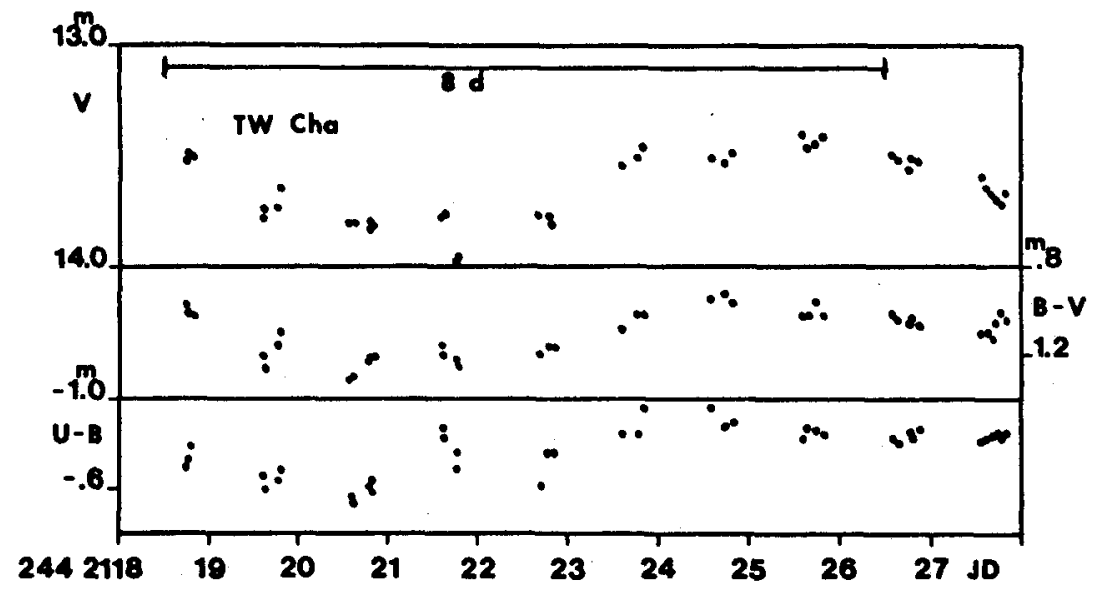

Figure 2: Light and colour curves of TW Cha

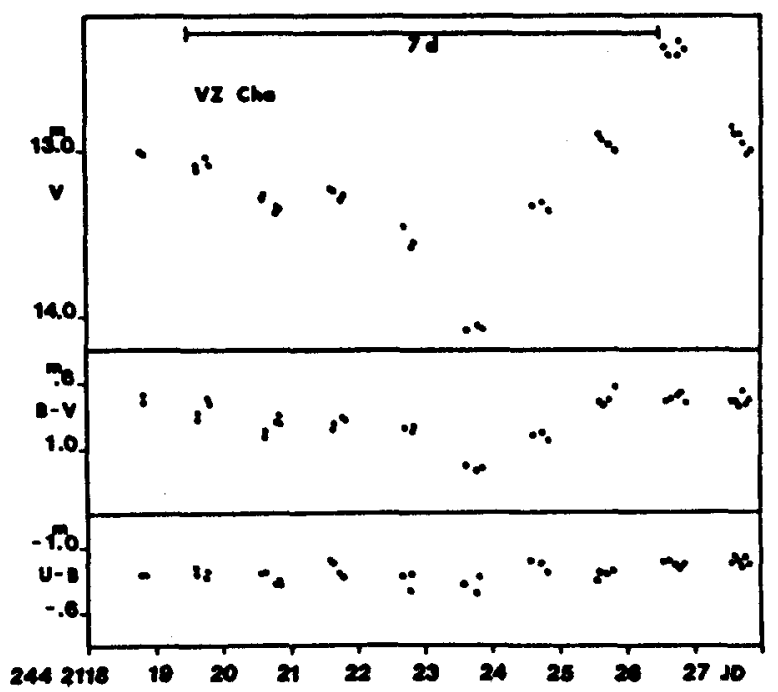

Figure 3: Light and colour curves of VZ Cha 
Some general features can be seen from the light and colour curves. The given periods represent the characteristic time scales between consecutive maxima and minima in the light and colour variations, with variable amplitude, well consistent with the results of the previous photographic survey by Mauder and Sosna (1975). The minima in the colour curves are clearly preceeding the minima in the visual light curves by one or two days for SY Cha and TW Cha. There is also evidence for small scale intrinsic activity during the individual nights, for instance at JD 2442121 in TW Cha or JD 2442125 in SY Cha. A large ultraviolett excess is found for all three stars, as to be expected in T Tauri variables. Grasdalen et al. (1975) studied the Chamaeleon T Association in the optical and infrared region. They found a reddening law for the dark cloud complex, the ratio of total to selective extinction being $R=5.5$, and derived a distance modulus of $(m-M){ }_{0}=5.3$ mag. The apparent $B-V$ colours are influenced by the excess radiation of the circumstellar envelope, due to the additional emission in the B band. From scarner observations in the optical and infrared, Grasdalen et al. were able to derive the influence of the ultraviolett continuum on the observed colours. Their results were used to correct the apparent colours for the ultraviolett excess, under the assumption, that the correction is the same for the whole cycle. Also, the apparent reddening and extinction, which they derived from their observations of $\mathrm{HD} 97300$ (AOV), were taken into account. The results are shown in a colour-magnitude-diagram in Figure 4, where the observations of a single night are combined to normal points. The very red intrinsic colours are well consistent with the low temperature features in the tracings of spectra obtained by Appenzeller (1977).

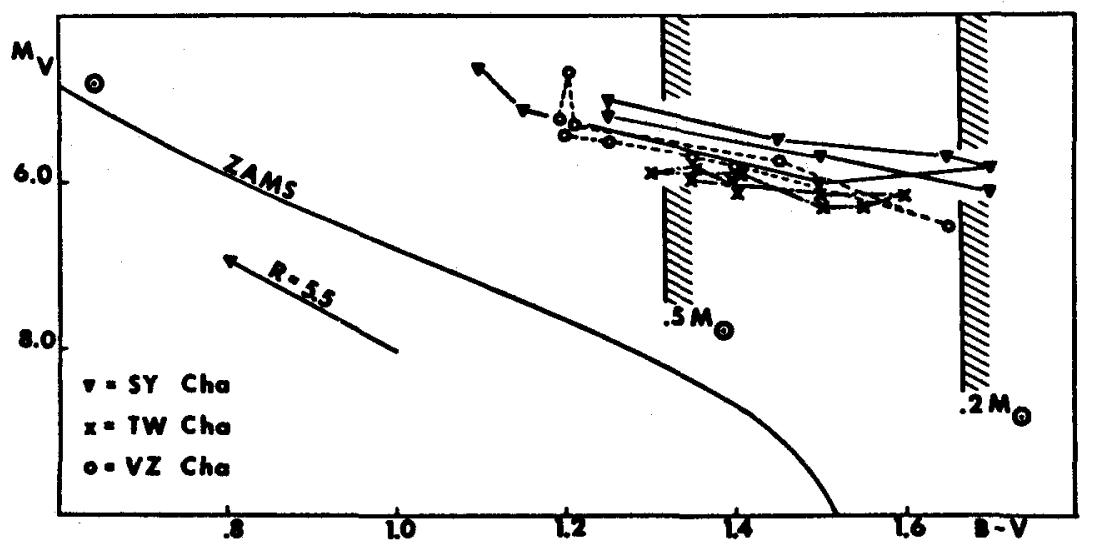

Figure 4: Cyclic variations of the three T Tauri stars in the HR-diagram. The vertical lines are Hayashi limits. 
For comparison, schematic Hayashi-lines are also given for stars of 0.5 and 0.2 solar masses, according to Iben (1965) and Grossmann and Graboske (1971), respectively. Of course, the transformation of bolometric to visual absolute magnitudes is very critical in this very low temperature part of the HR-diagram, but the main uncertainty is in the absolute magnitude, not in the colours. Therefore, no detailed limiting lines can be given. However, the approximate locations of the Hayashi limits point to remarkably low masses for the three T Tauri stars investigated here. It should be noted, that the Hayashi limits are valid only for stars in hydrostatic equilibrium. It is not clear, whether the T Tauri stars obey this condition. The quasicyclic behaviour of several T Tauri stars is not easy to understand. Poveda (1965) suggested that the cyclic part of the light variations with periods of several days could be due to clumps of planetesimals, orbiting in a disc around the I Tauri star. This explanation seems to be not very probable. The shape of the light curves of the present paper can hardly be explained by eclipses and the phase shift of the colour curves is not understood. Another explanation was proposed for instance by Hoffmeister (1965) who suggested, that the quasicyclic variations are due to the rotation of an active star. The photosphere of the $T$ Tauri star is assumed to be covered with large scale dark spots, causing a different net flux in the different directions. Thus, the period of the quasicycles should be identical with the rotational period of the star. No linear ephemeris are required since new spots can occur at different stellar longitudes. Though this model can explain the cyclical behaviour, it is difficult to understand the large armlitudes in the light and colour curves. Friedmann and currtler (1975) have calculated the light and colour variations of rotating spotted stars. According to their results, even in the most favourable cases a very large part of the star must be covered with spots to explain the observed light amplitudes. The amplitudes of the corresponding colour curves are much smaller than the observed ones and should be in phase with the light curves. We would like to propose still another possibility. The light and colour curves of SY Cha show a striking similarity with the respective curves of pulsating variables. The period-density-relation $P \cdot \sqrt{\rho / \rho_{0}}=Q$ would be satisfied for $Q=0.1$, which is a reasonable value for this part of the HR-diagram, if the mass is 0.2 solar masses and the radius is 6 solar radii. It is very difficult to compare the observed properties of low mass premain-sequence stars with theoretical calculations, since the photospheric conditions during the early evolutionary phases are highly uncertain, but the given figures are in the probable range of the early evolutionary tracks. Thus, it is not unreasonable to suggest pulsations with a period of several days for the three stars. Kippenhahn (1977) mentioned a possible mechanism which could drive the pulsations: the stars are still accreting matter onto their surfaces, the kinetic energy of the infalling material being converted into heat in a shock front. In a pulsating at- 
mosphere, the shock would be closer to the center of gravity at maximal contraction and consequently the kinetic energy would be larger. Thus, excess energy would be fed to the atmosphere at the right phase, eventually being sufficient to balance the damping in the stellar interior. Of course, numerical calculations are necessary to show, whether this effect is sufficient.

The author is indebted to I. Appenzeller for providing tracings of the spectra of SY Cha, TW Cha and VZ Cha. Helpful discussions with R. Kippenhahn and W. Tscharnuter are gratefully acknowledged.

\section{References:}

Appenzeller, I., 1977, private communication. Friedmann, C., Gürtler, J., 1975, Astron. Nachr. 296, 125

Grasdalen, G., Joyce, R., Knacke, R. F., Strom, S. E., Strom, K. M., 1975, A. J. 80,117

Grossmann, A. S., Graboske, H. C., 1971, Ap. J. 164,475

Henize, K. G., Mendoza, E. E., 1973, Ap. J. 180, 115

Hoffmeister, C., 1963, Veröff. Sonneberg 6,1

Eof fmeister, C., 1965, Veröff. Sonneberg 6 , 97

Tben, I., 1965, Ap. J. 141, 993

Kippenhahn, R., 1977, private communication

Mauder, H., Sosna, F. M., 1975, Inf. Bull. Var. Stars 1049

Poveda, A., 1965, Bol. Obs. Tonantzintlay Tacubaya $\underline{4}, 15$

\section{I S C U S S I O N of paper by MAUDER:}

KIPPENHAHN: The low masses for the $T$ Tau stars you derive by comparing your observations with models on the Hayashi track. But these are models which are in hydrostatic equilibrium and which have surface regions which are thermally adjusted. If, like in Larson's picture, there is no hydrostatic equilibrium and if the infalling material releases energy near the surfaces while you are looking through an almost opaque envelope, you can have objects in the region of the HR diagram which is beyond the Hayashi line.

MAUDER: Yes, I agree completely with your remark. However, I feel that it is not very probable to have several stars of higher mass at this extremely red, low Iuminosity part of the HR-Diagram; of course, this is only a qualitative statement.

KRAFT: How did you make the transformation from $\log \mathrm{T}_{\mathbf{e}}$ (at the Hayashi limit) to (B-V)? This actually determines your theoretical masses, and it is very sensitive to the ratio of mixing length to scale-height.

MAUDER: I did not use the theoretical limiting lines for a determination of the masses. The only thing that can be said is, that the stars under consideration are mainly in the very low mass domain of the HR-Diagram. This is not a quantitative mass determination, but only a qualitative result. 
R.N. THOMAS: If I understand well, masses come only from model calculations? Do you have any empirical confirmation?

MAUDER: Indeed, the Hayashi-Cameron-limits are theoretical lines; no attempt was made to derive masses from the empirical data.

KOPAL: Wouldn't you say that observational selection should favor discovery of $T$ Tauri stars of low mass (because of their longer life-time?

MAUDER: As I think, the low mass $T$ Tauri stars are also the less Iuminous ones. 